\title{
Volumetric evaluation of fat in the renal sinus in normal subjects using stereological method on computed tomography images and its relationship with body composition
}

\author{
V. Caglar ${ }^{1}$, A. Songur², M. Acar ${ }^{3}$, R. Uygur ${ }^{1}$, O.A. Alkoc ${ }^{4}$, T. Acar ${ }^{5}$ \\ ${ }^{1}$ Department of Anatomy, Faculty of Medicine, Namık Kemal University, Tekirdag, Turkey \\ ${ }^{2}$ Department of Anatomy, Faculty of Medicine, Afyon Kocatepe University, Afyonkarahisar, Turkey \\ ${ }^{3}$ Department of Radiology, Faculty of Medicine, Istanbul Medeniyet University, Istanbul, Turkey \\ ${ }^{4}$ Department of Anatomy, Faculty of Medicine, Istanbul Medeniyet University, Istanbul, Turkey \\ ${ }^{5}$ Department of Anatomy, Faculty of Medicine, Sifa University, Izmir, Turkey
}

[Received 27 December 2013; Accepted 28 January 2014]

Background: The aim of the study was: to describe a simple, accurate and practical technique for estimating the volume of adipose tissue within the renal sinus (RS) using stereological method on computed tomography (CT) images; to establish a population database for volume of fat within the RS from 21 to 80 years of age; to investigate the effect of age, gender, body mass index (BMI) and abdominal diameters on RS fat volume in normal subjects.

Materials and methods: We retrospectively reviewed abdominal CT examinations of 240 patients without renal pathology between the ages of 21 and 80 years. There were 6 groups of patients, with 40 patients for each decade.

Results: RS fat volumes in the left and right kidney were $5.70 \pm 2.87 \mathrm{~cm}^{3}$ and $4.15 \pm 2.39 \mathrm{~cm}^{3}$, respectively, in males and $3.51 \pm 2.67 \mathrm{~cm}^{3}$ and $2.49 \pm 2.16 \mathrm{~cm}^{3}$, respectively, in females. $R S$ fat volume and age were positively correlated for both kidneys (left: $r=0.46$; right: $r=0.44 ; p<0.001$, both), though it appeared to decline after age 70.

Conclusions: Quantitative data may allow clinicians to better estimate the age-related RS fat volume changes and help them in decision making. (Folia Morphol 2014; 73, 3: 302-308)

Key words: age related changes, computed tomography, fat volume, renal sinus, stereology

\section{INTRODUCTION}

The renal sinus (RS) is a space that forms the medial border of the kidney and is surrounded by the renal parenchyma laterally. The renal arteries, veins, lymphatic vessels, nerve fibres, renal pelvis and major and minor calices are located within the RS.
Varying amounts of adipose tissue envelops these structures within the RS [5]. This area may be affected by various pathologic conditions arising from the different tissues within the RS [19].Tumorous and nontumorous lesions can occur in RS. These lesions include lipomatosis, RS cysts, vascular lesions and

Address for correspondence: Dr V. Caglar, Department of Anatomy, Namık Kemal University Faculty of Medicine, Tekirdag, 59100 Turkey, tel: +90 507387 7279, +90 282250 5523, telefax: +90 28225099 28, e-mail: vcaglar32@hotmail.com

The manuscript has been presented at a congress ( $4^{\text {th }}$ International Symposium of Clinical and Applied Anatomy) as a poster at June 28 - July 01, 2012, Ankara, Turkey. 
fluid collections. Observation of RS fat is important in detecting small tumours and determining the tumour stage [22]. Human studies and animal models have demonstrated fat accumulation in the RS $[5,10,14$, 17]. The accumulation of RS fat is important because the renal vein and artery pass through the RS and may be compressed by adipose tissue [11]. Animal models have described excessive accumulation of fat within the RS and this accumulation displaces and compresses the low pressure renal lymphatics, veins and the ureters $[2,7,9,10]$. Fat within the RS may have an effect on hypertension and cardiovascular risk through one of several mechanisms $[10,11]$. Compression of structures within the RS increases renal hydrostatic pressure and activates the renin-angiotensin-aldosterone system (RAAS) [10, 21]. Activation of the RAAS promotes hypertension, insulin resistance, atherosclerosis, and other adverse physiological effects related to obesity $[15,21]$. Renal lipotoxicity and its role in the pathogenesis of renal disease are not fully understood, but it has been assumed that renal disease progression is promoted by the accumulation of fat in the kidneys [24-26]. Here, we describe a simple, accurate and practical technique for estimating the volume of adipose tissue within the RS volume without changing the routine computed tomography (CT) imaging scanning procedure. In addition, our aim in this study was to establish a population database for volume of fat within the RS from age 21 to 80 years by the stereological methods and to investigate the effect of age, gender, body mass index (BMI) and sagittal abdominal diameter (SAD) and transvers abdominal diameter (TAD) on RS fat volume in normal subjects. Furthermore, the fat volume of the RS has been the subject of many studies, none of which has used stereological methods to estimate this volume.

\section{MATERIALS AND METHODS}

The study was conducted in compliance with the Helsinki Declaration. Patients who were referred to Radiology Department for various reasons for abdominopelvic CT scan between 2009 and 2010 were included in this study. CT scans of 643 patients were examined retrospectively. Abdominal CT examinations of 240 adults ( 120 men and 120 women; mean age $50 \pm 17$ and $49 \pm 17$ years, respectively) were included in this study. Age, body weight [kg] and height [m] of each patient were recorded at the time of their CT scan. Furthermore the backgrounds of patients, vascular disease, hypertension, diabetes mellitus and a history of renal surgery were recorded at the time of CT scan. To avoid measurement errors, patients with known vascular disease, hypertension, diabetes mellitus and with a history of renal surgery were excluded from the study. Also, patients with hydronephrosis, renal cysts, renal artery stenosis or renal malformation were excluded from study. CT scans were performed with a 6 detector-row multi-detector CT scanner (Philips Brilliance 6, Philips Medical Systems, Amsterdam, The Netherlands) after administration of oral (composed of 1,000 mL of water and $250 \mathrm{~mL}$ of lactulose; Osmolac, Biofarma, Istanbul, Turkey) and intravenous contrast material $(1.6 \mathrm{~mL} / \mathrm{kg}$ body weight, rate $3 \mathrm{~mL} / \mathrm{s}$, delay $65 \mathrm{~s}$; Omnipaque 350, iohexol, GE Healthcare, Ireland), and reconstructed to contiguous $5 \mathrm{~mm}$ transverse sections. Participants were selected so that was equal representation of sex and 10-year age groups (21-30, 31-40, 41-50, 51-60, 61-70 and 71-80 years old). BMI values were classified according to World Health Organisation criteria. The subjects were classified into lean $(n=87, B M I 18.5 \leq$ $\left.\leq 24.9 \mathrm{~kg} / \mathrm{m}^{2}\right)$, overweight $\left(\mathrm{n}=101,25 \mathrm{~kg} / \mathrm{m}^{2} \leq \mathrm{BMI}<\right.$ $\left.<29.9 \mathrm{~kg} / \mathrm{m}^{2}\right)$, and obese $(\mathrm{n}=48,30 \leq \mathrm{BMI} \geq$ $\geq 39.9 \mathrm{~kg} / \mathrm{m}^{2}$ ) groups. We examined possible differences in the RS fat volume among these groups.

\section{Cavalieri principle estimation of the volume of fat within the RS}

RS fat accumulation was measured using Cavalieri principle of stereological methods on abdominal CT scans. Pixel density in Hounsfield units (HU) was used to identify adipose tissue based on a window width of -100 to $-20 \mathrm{HU}$ [4].

Cavalieri principle requires sectioning the structure with a series of parallel planes. An unbiased estimate of volume can be obtained by multiplying the total area of the section cut surfaces through the structure on all the sections. The point counting grid, which has some point sets at distinct densities on a transparent sheet, can be used to estimate the cut surface area of the sections. The point counting method consists of overlying each selected section with a regular grid of test points, which is randomly positioned. Counting procedure should be superimposed on the section three times and the mean number of points hitting the objects should be used to estimate cut surface area of the section. A point is a (+) shaped lines and it is said to hit the object, if the upper right hand corner of the intersection of the cross lines representing them on the test system lies inside the 


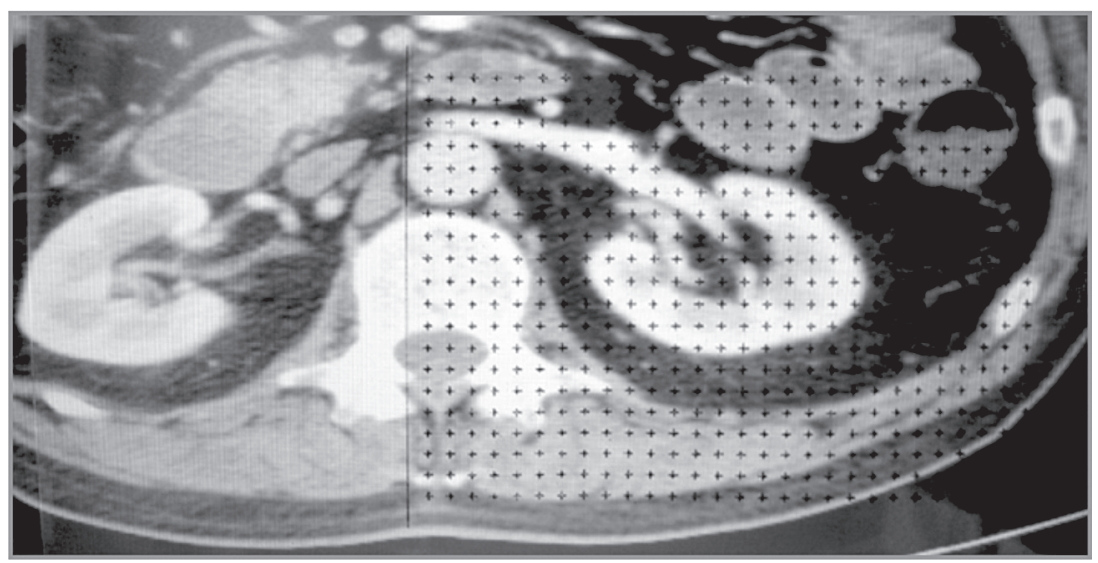

Figure 1. A renal sinus slice section in axial plane, a point counting grid superimposed on the computed tomography scan.

object. After each superimposition, the number of test points hitting the structure of interest on the sections is counted.

A series of the abdominal CT images of $5 \mathrm{~mm}$ thick sections were used to estimate the volume of fat within the RS. The CT images were downloaded from Picture Archiving and Communication System (PACS) (Enlil, Eskisehir, Turkey) and transferred to a personal computer (PC). Studies were viewed on PC monitor to review digital imaging and communications in medicine (DICOM) header information (age, sex, display field of view, and section thickness), which was subsequently recorded in a spreadsheet program. A square grid system with $\mathrm{d}=0.15 \mathrm{~cm}$ were placed randomly on each cross section CT images of RS and the points hitting the surface area of RS were counted for each section (Fig. 1). The medial border of the renal sinus was enclosed by extending a straight line tangential to each hilar lip. Counting procedure was performed 3 times for each image of a cross-section and averages of these counts were recorded. Points corresponding to the boundaries of the RS area were included in the count. Other points outside the boundaries, even if they were very close, were not included in count. After initial setup and preparation of the formula, the point counts and other data were entered for each RS fat volume and results were obtained automatically.

$$
V=t \times[(S U \times d) / S L]^{2} \times \Sigma P
$$

Calculations were carried out as follows; ' $t$ ' represents the section thickness of consecutive sections,
'SU' - the scale unit of the CT images, ' $d$ ' - the distance between the test points of the grid, 'SL' the measured length of the scale sectioned on the $C T$ images, and ' $\Sigma P$ ' is the total number of markers corresponding to the fat within RS $[3,23]$.

\section{Anthropometric measurements}

On CT images, SAD was measured as the distance between the anterior and posterior skin on one cross-sectional scan obtained at the level of the fourth lumbar vertebra [13, 27]. TAD was measured on the outer borders of the body outline from the right side to the left side at the widest point and the same vertebral level $[3,13]$.

\section{Statistical analysis}

All statistical analyses were performed using the SPSS 11.0 for Windows. All parametric results were expressed as mean \pm standard deviation for each group. The variances of the groups (homogeneity) was assessed by Levene's test. The paired t-test or Mann-Whitney $\mathrm{U}$ and ANOVA or Kruskal Wallis $\mathrm{H}$ test was used for the analysis of the groups. Duncan test was used to compare group means. Correlation was determined using the Pearson correlation coefficient. Differences were considered significant if two-tail $p$ values were less than 0.05 .

\section{RESULTS}

Participating males and females were similar in age, BMI and SAD. TAD was larger in females and the CT measured in left and right kidney RS fat volumes were larger in males. Patient demographics and RS fat volume in adults is shown in Table 1. 
Table 1. Characteristics of subjects enrolled in computed tomography imaging studies of the renal sinus (RS)

\begin{tabular}{lcccc}
\hline & Total & Female & Male & P* $^{*}$ \\
\hline $\mathrm{N}$ & 240 & 120 & 120 & $<0.001$ \\
RS fat volume $\left(\right.$ left kidney, $\left.\mathrm{cm}^{3}\right)$ & $4.61 \pm 2.97$ & $3.51 \pm 2.67$ & $5.70 \pm 2.87$ & $<0.001$ \\
RS fat volume $\left(\right.$ right kidney, $\left.\mathrm{cm}^{3}\right)$ & $3.32 \pm 2.42$ & $2.49 \pm 2.16$ & $4.15 \pm 2.39$ & NS \\
Age [years] & $49.95 \pm 17.46$ & $49.81 \pm 17.68$ & $50.09 \pm 17.31$ & NS \\
Body mass index $\left[\mathrm{kg} / \mathrm{m}^{2}\right]$ & $26.99 \pm 4.96$ & $27.81 \pm 5.92$ & $26.18 \pm 3.61$ & NS \\
Sagittal abdominal diameter $[\mathrm{cm}]$ & $23.33 \pm 4.22$ & $23.29 \pm 4.62$ & $23.36 \pm 3.81$ & 0.043 \\
Transverse abdominal diameter $[\mathrm{cm}]$ & $33.47 \pm 4.12$ & $34.1 \pm 4.83$ & $32.86 \pm 3.51$ & \\
\hline
\end{tabular}

Values are means \pm standard deviation; ${ }^{*} p$ value shows comparison of men and women; NS — non significant $(p>0.05) ; n$ - the number of participants in each group

\section{Effect of age on the RS fat volume}

There was no statistically significant difference in age values between males and females. There was no statistically significant difference between RS fat volume increase in the second, third and fourth decades in both kidneys of total group, but there was significant difference in sixth and seventh decades. The RS fat volume in the right and left kidney continues to increase until the age of 70 and then declines thereafter. The RS fat volume reaches a maximum in the seventh decade in both kidneys $\left(4.95 \mathrm{~cm}^{3}\right.$ for right and $6.73 \mathrm{~cm}^{3}$ for left kidney) (Fig. 2). In the total study population, we found significant difference between RS fat volume of the right and left kidney, except for the sixth decade. There was a correlation $(p<0.001$, $r=0.439$ for right kidney and $p<0.001, r=0.461$ for left kidney) in the total study population between the RS fat volume in both kidneys and the age (Table 2).

The RS fat volume increases in each decade in both kidneys in female group were similar to that observed in the total study population. But there weres significant differences in sixth and seventh decades. The RS fat volume reaches a maximum in the seventh decade in both kidneys $\left(4.81 \pm 2.12 \mathrm{~cm}^{3}\right.$ for right

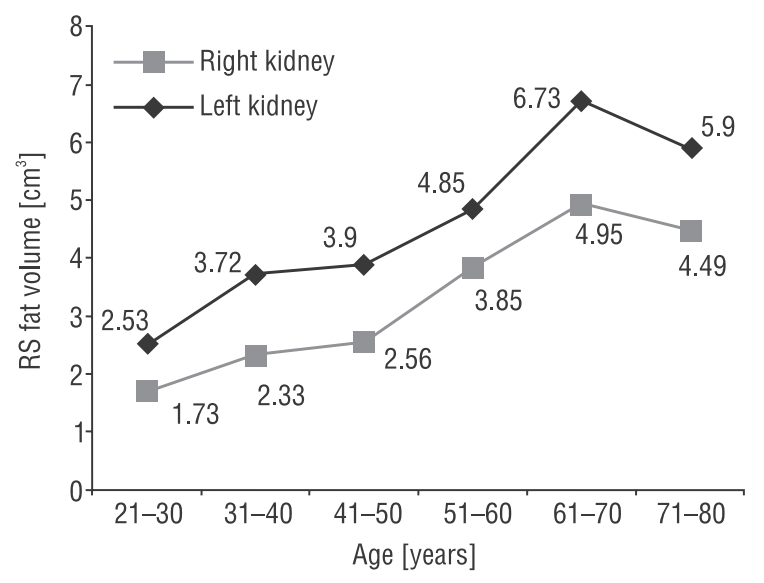

Figure 2. Renal sinus (RS) fat volume are shown for each decade of total group. Values are means \pm standard deviation.

and $7.12 \pm 3.17 \mathrm{~cm}^{3}$ for left) and then declines thereafter (Fig. 3). In female group ( $p<0.001, r=0.489$ for right kidney and $p<0.001, r=0.484$ for left kidney) we found correlation between the RS fat volume in both kidneys and the age.

RS fat volume increases in the second and third decades in both kidneys in male group were similar to that in female group and the total study popula-

Table 2. Relationship between the parameters used and renal sinus (RS) fat volume for total study population

\begin{tabular}{lccccc}
\hline The parameters & \multicolumn{2}{c}{ RS fat volume of left kidney $(\mathbf{n}=\mathbf{2 4 0})$} & & RS fat volume of right kidney $(\mathbf{n}=\mathbf{2 4 0})$ \\
\cline { 2 - 3 } & $\mathbf{r}$ & $\mathbf{p}$ & & $\mathbf{r}$ & $\mathbf{p}$ \\
\hline Age [years] & 0.439 & 0.001 & & 0.461 & $<.001$ \\
Body mass index $\left[\mathrm{kg} / \mathrm{m}^{2}\right]$ & 0.181 & 0.005 & & 0.224 & $<0.001$ \\
Sagittal abdominal diameter $[\mathrm{cm}]$ & 0.384 & $<0.001$ & & 0.407 & $<0.001$ \\
Transverse abdominal diameter $[\mathrm{cm}]$ & 0.272 & $<0.001$ & & 0.275 & $<0.001$ \\
\hline
\end{tabular}

$r$ - value is the correlation coefficient; $p-$ value is the probability 


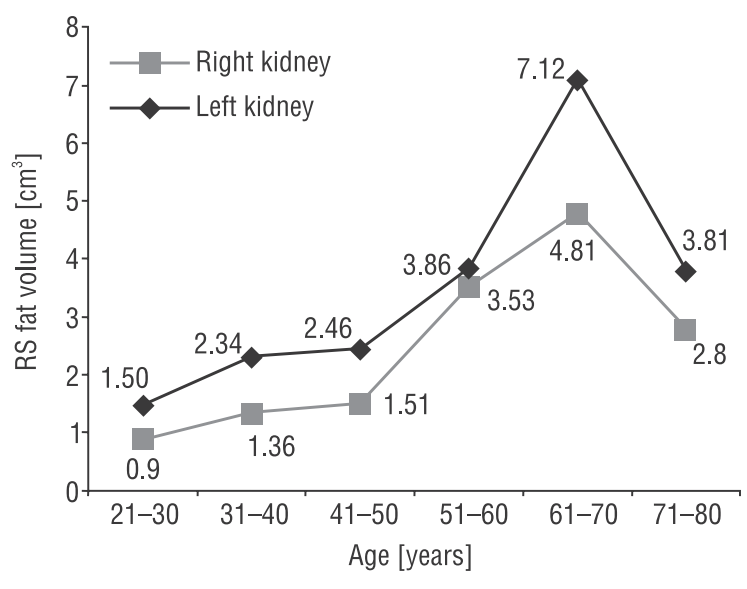

Figure 3. Renal sinus (RS) fat volume are shown for each decade of female group. Values are means \pm standard deviation.

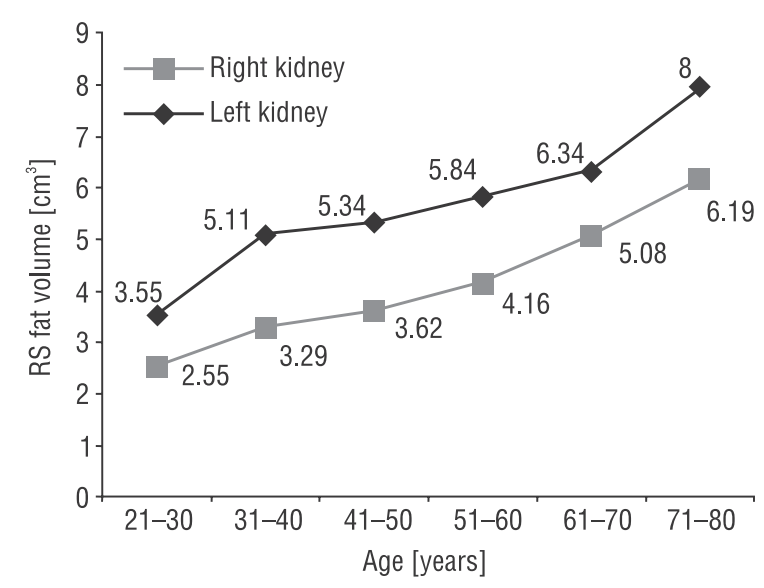

Figure 4. Renal sinus (RS) fat volume are shown for each decade of male groups. Values are means \pm standard deviation.

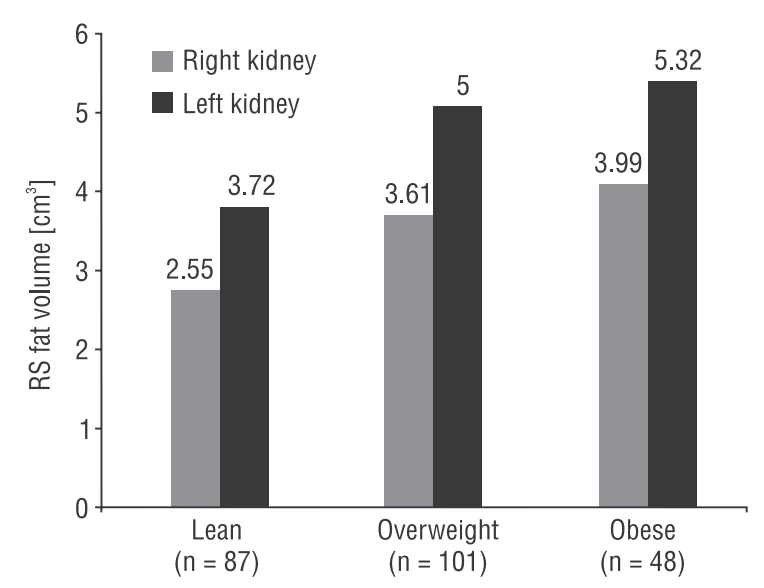

Figure 5. Comparison of renal sinus (RS) fat volume according to body mass index groups. Values are means \pm standard deviation; $\mathrm{n}-$ the number of participants in each group. tion. From age 40 to 80 years, RS fat volume reaches a plateau $\left(6.19 \pm 1.69 \mathrm{~cm}^{3}\right.$ for right and $8 \pm 2.23 \mathrm{~cm}^{3}$ for left) (Fig. 4). In male groups ( $p<0.001, r=0.451$ for right kidney and $p<0.001, r=0.493$ for left kidney) we found correlation between the RS fat volume in both kidneys and the age.

\section{Effect of gender on the RS fat volume}

There was no correlation between age and BMI in male and female groups of participants. We found that the RS fat volume in males was $4.15 \mathrm{~cm}^{3}$ for right and $5.7 \mathrm{~cm}^{3}$ for left kidney. The RS fat volume in females was $2.49 \mathrm{~cm}^{3}$ for right and $3.51 \mathrm{~cm}^{3}$ for left kidney. The RS fat volume of both kidneys in males was significantly bigger than that of the females $(p<0.001)$ (Table 1).

\section{Effect of BMI on the RS fat volume}

Based on BMI, the subjects were identified as lean, overweight and obese. The effects of obesity on RS fat volume were examined. RS fat volume in left kidney was 25.6 and $30 \%$ greater in the overweight and obese groups compared with the lean group $\left(3.72 \pm 2.59,5 \pm 2.92\right.$, and $5.32 \pm 3.33 \mathrm{~cm}^{3}$ in lean, overweight, and obese groups, respectively). RS fat volume in right kidney was 29.3 and $36 \%$ greater in the overweight and obese groups compared with the lean group $(2.55 \pm 1.89,3.61 \pm 2.44$, and $3.99 \pm$ $\pm 2.91 \mathrm{~cm}^{3}$ in lean, overweight, and obese groups, respectively). There was a correlation between the left and right kidney RS fat volumes and BMI $(r=0.181$, $p=0.005$ and $r=0.224, p<0.001$, respectively) (Fig. 5). Comparison of RS fat volume according to $\mathrm{BMI}$ groups showed significant difference between lean and obese groups but no significant difference between overweight and obese groups.

\section{Effect of abdominal diameters on the RS fat volume}

In our study, we found a correlation between the RS fat volume in both kidneys and SAD and TAD for total study population (Table 2).

\section{DISCUSSION}

Lipid accumulation in the kidney is considered to be the effect of progression of kidney disease [11, 24, 25]. Foster et al. [11] reported that the highest prevalence of chronic kidney disease was observed among individuals with fatty kidney (RS fat $\geq 0.445 \mathrm{~cm}^{2}$ in women and $\geq 0.710 \mathrm{~cm}^{2}$ in men). Moreover, individuals with fatty kidney had higher imputed systolic and diastolic 
blood pressures compared with those without fatty kidney [11]. Chughtai et al. [5] reported that RS fat volume is associated with the number of prescribed antihypertensive medications and stage II hypertension. In animal models of diet-induced obesity, intrarenal fat deposits can directly or indirectly alter renal structure $[10,17]$. For example, diet-induced obesity in dogs and rabbits is associated with larger kidneys and increased fat deposits in the RS [10]. The RS fat accumulation leads to increases in renal interstitial pressure through the compression of the renal vein and lymph vessels exiting the kidney [17].

RS fat volume may have both diagnostic and prognostic benefits, but the absence of normal data and lack of knowledge on factors that affect it has restricted its use. We have established population data for RS fat volumes among the people from 21 to 80 years. We also observed the fact that RS fat volume measurements are correlated with parameters such as age, BMI, gender. Moreover since SAD and TAD are considered a good indicators of the amount of visceral adipose tissue, we examined the relationship between SAD, TAD and the RS fat volume. The anthropometric results may be a good indicator of RS fat volume. Various imaging modalities can be used for examination of the RS, such as ultrasonography, $\mathrm{CT}$, magnetic resonance imaging and angiography. $\mathrm{CT}$ is the most sensitive, efficient, and comprehensive imaging modality for examination of RS [19, 22]. Therefore, in this study CT images were used for volume measurements of RS.

There are several packages developed for volume estimation, for example, Analyse and Image J. This software has region of interest function based on manual techniques. Manual techniques, including planimetry or tracing methods that require the investigator to delineate the RS region based on reliable anatomical landmarks, whilst the software package provides information on volume. The effect of physician education and professional experience on the use of planimetry or tracing methods are significant. For this reason evaluation of the volume may lead to subefectivity. Moreover, these techniques are still time consuming, costly and are not accepted in clinical practice. Thus, the point-counting technique used in this and previous studies makes volume estimation easier and quicker than manual techniques [1, 3].

We report that the RS fat volume increases in linear fashion from the age of 21 to 70 and declines thereafter. Dietrich and Kangarloo [8] reported the increased adipose tissue in the RS with age in 58 chil- dren aged 1 day to 17 years. Our study, in accordance with previous studies, has shown a decline in kidney size only after the age of $60[16,20]$. RS fat volume measurements in both kidneys were correlated with the subjects' age (right kidney: $r=0.439, p<0.001$, left kidney: $r=0.461 p<0.001)$. In all age groups except for the sixth decade, the RS fat volume of the right kidney was significantly smaller than that of the left. In female and male groups, except for sixth and seventh decades, the RS fat volume of the right kidney was significantly smaller than that of the left. These results were consistent with the results found by Cohen et al. [6] and opposed to the results found by Muto et al. [18]. These differences in the results may be due to between-population heterogeneity. We report that the amount of fat in the RS normally and gradually increases with age.

The results of this study also clarify the influence of gender on kidney size. In the present study, there were significant differences in the RS fat volumes of both kidneys between males and females $(p<0.001)$. This result is consistent with previous measurements of renal volume $[12,18]$. Johnson et al. [12] found that RS fat volume varies inversely with the volume of parenchyma. This result suggests that fat accumulation in RS causes changes in parenchymal volume size. Miletic et al. [16] reported that there was no effect of gender on renal size when differences in body size were taken into account. This result may be due to insufficient sensitivity of the measurements of parenchymal tissue.

Our study also examined the effect of BMI on RS fat volume in adults. Although RS fat accumulation usually correlates with body height and weight [22] the relationship between RS fat volume and BMI has not yet been elucidated. In this study RS fat volume measurements in both kidneys were correlated with the subjects' BMI, SAD and TAD. We also found more RS fat volume in obese individuals. The presence of a significant relationship between BMI and RS fat volume suggests an effect of obesity on the RS fat volume. In the literature on animals, RS fat accumulation has been described in obesity $[6,24]$. Dwyer et al. [10] reported that in obese rabbits RS mass was $61 \%$ and obesity alone can cause renal lipomatosis. Rha et al. [22] reported that increase in fat amount in RS involves prominent fat proliferation that leads to mass effect on the intrarenal collecting system. We reported that the quantity of fat in the RS normally and gradually increases with obesity. This increased 
volume of fat in RS may be sufficient to externally compress renal veins and lymphatics. Furthermore, in obese individuals, RS fat accumulation may contribute to arterial hypertension via restriction of blood flow.

\section{CONCLUSIONS}

We have established a population data for RS fat volumes among subjects aged from 21 to 80 years. We also reported that RS fat volume normally and gradually increases with age and BMI. Moreover, we report that the RS fat volumes are greater in males than in females, and RS fat volume of the right kidney was significantly smaller than that of the left one. In addition, the findings of the present study performed using the stereological methods could provide data for the evaluation of normal and pathological values of the fat volume in the RS.

\section{REFERENCES}

1. Acer N, Sahin B, Baş O, Ertekin T, Usanmaz M (2007) Comparison of three methods for the estimation of total intracranial volume: stereologic, planimetric, and anthropometric approaches. Ann Plast Surg, 58: 48-53.

2. Bobulescu IA, Dubree M, Zhang J, McLeroy P, Moe OW (2008) Effect of renal lipid accumulation on proximal tubule $\mathrm{Na}+/ \mathrm{H}+$ exchange and ammonium secretion. Am J Physiol Renal Physiol, 294: 1315-1322.

3. Caglar V, Songur A, Yagmurca M, Acar M, Toktas M, Gonul Y (2012) Age-related volumetric changes in pancreas: a stereological study on computed tomography. Surg Radiol Anat, 34: 935-941.

4. Choyke PL (2006) Radiologic imaging of renal cell carcinoma: its role in diagnosis. In: Vogelzang NJ, Scardino PT, Shipley WU, Debruyne FMJ, Linehan WM eds. Comprehensive textbook of genitourinary oncology. $3^{\text {rd }}$ Ed. Lippincott Williams and Wilkins, Philadelphia, USA, pp. 709-723.

5. Chughtai $\mathrm{HL}$, Morgan TM, Rocco M, Stacey $B$, Brinkley TE, Ding J, Nicklas B, Hamilton C, Hundley WG (2010) Renal sinus fat and poor blood pressure control in middle-aged and elderly individuals at risk for cardiovascular events. Hypertension, 56: 901-906.

6. Cohen El, Kelly SA, Edye M, Mitty HA, Bromberg JS (2009) MRI estimation of total renal volume demonstrates significant association with healthy donor weight. Eur J Radiol, 71: 283-287.

7. Deji N, Kume S, Araki S, Soumura M, Sugimoto T, Isshiki K, ChinKanasaki M, Sakaguchi M, Koya D, Haneda M, Kashiwagi A, Uzu T (2009) Structural and functional changes in the kidneys of high-fat diet-induced obese mice. Am J Physiol Renal Physiol, 296: 118-126.

8. Dietrich RB, Kangarloo H (1986) Kidneys in infants and children: evaluation with MR. Radiology, 159: 215-221.

9. do Carmo JM, Tallam LS, Roberts JV, Brandon EL, Biglane J, da Silva AA, Hall JE (2009) Impact of obesity on renal structure and function in the presence and absence of hypertension: evidence from melanocortin-4 receptor-deficient mice. Am J Physiol Regul Integr Comp Physiol, 297: R803-R812.
10. Dwyer TM, Mizelle HL, Cockrell K, Buhner P (1995) Renal sinus lipomatosis and body composition in hypertensive, obese rabbits. Int J Obes Relat Metab Disord, 19: 869-874.

11. Foster MC, Hwang SJ, Porter SA, Massaro JM, Hoffmann U, Fox CS (2011) Fatty kidney, hypertension, and chronic kidney disease: the framingham heart study. Hypertension, 58: 784-790.

12. Johnson S, Rishi R, Andreea A, Khawandi W, Al-Said J, Miller NG, Lin E, Baumgarten DA, O'Neill WC (2011) Determinants and functional significance of renal parenchymal volume inadults. Clin J Am Soc Nephrol, 6: 70-76.

13. Kvist H, Chowdhury B, Grangard U, Tylen U, Sjostrom L (1988) Total and visceral adipose-tissue volumes derived from measurements with computed tomography in adult men and women: predictive equations. Am J Clin Nutr, 48: 1351-1136.

14. Lee HS, Lee JS, Koh HI, Ko KW (1991) Intraglomerular lipid deposition in routine biopsies. Clin Nephrol, 36: 67-75.

15. Manrique C, Lastra G, Gardner M, Sowers JR (2009) The renin-angiotensin-aldosterone system in hypertension: roles of insulin resistance and oxidative stress. Med Clin North Am, 93:569-582.

16. Miletic D, Fuckar Z, Sustic A, Mozetic V, Stimac D, Zauhar G (2009) Sonographic measurement of absolute and relative renal length in adults. J Clin Ultrasound, 26: 185-189.

17. Montani JP, Carroll JF, Dwyer TM, Antic V, Yang Z, Dulloo AG (2004) Ectopic fat storage in heart, blood vessels and kidneys in the pathogenesis of cardiovascular diseases. Int J Obes Relat Metab Disord, 28: 58-65.

18. Muto NS, Kamishima T, Harris AA, Kato F, Onodera Y, Terae $S$, Shirato H (2011) Renal cortical volume measured using automatic contouring software for computed tomography and its relationship with $\mathrm{BMI}$, age and renal function. Eur J Radiol, 78: 151-156.

19. Nikolaidis $P$, Gabriel $H$, Khong $K$, Brusco M, Hammond $N$, Yagmai V, Casalino D, Hoff F, Patel S, Miller F (2008) Computed tomography and magnetic resonance imaging features of lesions of the renal medulla and sinus. Curr Probl Diagn Radiol, 37: 262-278.

20. Nyengaard JR, Bendtsen TF (1992) Glomerular number and size in relation to age, kidney weight, and body surface in normal men. Anat Rec, 232: 194-120.

21. Ott CE, Navar LG, Guyton C (1971) Pressures in static and dynamic states from capsules implanted in the kidney. Am J Physiol, 221: 394-400.

22. Rha SE, Byun JY, Jung SE, Oh SN, Choi YJ, Lee JM (1995) The renal sinus: pathologic spectrum and multimodality imaging approach. Radiographics, 24: 117-131.

23. Sahin B, Emirzeoglu M, Uzun A, Incesu L, Bek Y, Bilgic S, Kaplan S (2003) Unbiased estimation of the liver volume by the Cavalieri principle using magnetic resonance images. Eur J Radiol, 47: 164-170.

24. Sijens PE, Edens MA, Bakker SJL, Stolk RP (2010) MRI-determined fat content of human liver, pancreas and kidney. World J Gastroenterol, 16: 1993-1998.

25. Wang IK, Wang ST, Chang HY, Lin CL, Kuo HL, Chen TC, Lee CH, Chuang FR (2005) Prognostic value of acute physiology and chronic health evaluation II and organ system failure in patients with acute renal failure requiring dialysis. Ren Fail, 27: 663-669.

26. Weinberg JM (2006) Lipotoxicity. Kidney Int, 70: 1560-1566 .

27. Zaidi E, Daly B (2006) CT and clinical features of acute diverticulitis in an urban u.s. population: rising frequency in young, obese adults. AJR, 187: 689-694. 